\title{
A simple, inexpensive stimulus waveform generator
}

\author{
RANDALL K. EPPERSON and ALAN J. WILSON \\ Southern College of Optometry, Memphis, Tennessee
}

\begin{abstract}
A simple circuit for a sine wave generator is described. The total cost for construction is approximately $\$ 30.00$. At present, the unit is used to generate a sinusoidally modulated light stimulus for steady state visual evoked response experiments. Modifications of the circuit for other applications are discussed.
\end{abstract}

The unit is constructed with two integrated circuits (ICs) and 17 discrete components. Figure 1 is a schematic diagram of the circuit. IC1 is an INTERSIL ICL8038 precision waveform generator voltage-controlled oscillator. In our application, this device generates all the signals necessary for the visual evoked response instrumentation of our laboratory - that is, a sine wave for modulating an array of light-emitting diodes (LEDs), and a square wave to trigger our averaging computer. The ICL8038 can operate over a range of from less than $.001 \mathrm{~Hz}$ to more than $1 \mathrm{MHz}$. A triangle waveform is also available. The ICL8038 features high-level outputs, low distortion, high linearity, and a variable duty cycle, all of which make it cost effective for a variety of applications.

IC2 is a National Semiconductor Corporation (1982) LM124 low-power quad operational amplifier. IC2a, in conjunction with resistor $\mathrm{Rb}$, provides a means of adjusting the amplitude of the sine wave. The variable resistor in the feedback loop of this section limits the maximum amplitude of the sine wave in order to prevent overdriving the following stages.

IC2b and IC2c function together in a dc-restoration configuration. This permits operation of our LED load above cutoff in the most linear portion of its operating curve. When the signal at the inverting input of IC2b swings below the voltage level at the noninverting input (set by adjusting Rc for the desired offset), the op amp allows diode D1 to turn on, thus forming a low-impedance path to charge capacitor $\mathrm{C} 2$. After the diode is turned off, the charge is retained on the capacitor. This action gives the circuit a fast-attack/slow-decay response to input-level changes. Note that D1 should be a germanium diode in order for the circuit to operate properly. Note also that, for operation at frequencies higher than approximately $125 \mathrm{~Hz}$, the value of $\mathrm{C} 2$ should be reduced. Experimentation will point to the best value for the frequency range to be used. By reversing the diode, it is possible to clamp

The authors' mailing address is: Visual Perception Laboratory, Southern College of Optometry, Memphis, TN 38104. positive peaks, should this be necessary in other applications.

IC2c is a unity-gain buffer that drives output transistor Q1. In our application, the load on Q1 is an array of 75 LEDs connected in parallel.

\section{CONSTRUCTION AND OPERATION}

Layout and wiring of the components of this unit are not critical. Our prototype was constructed on a small piece of perfboard using wire-wrap techniques. It is housed in an instrumentation cabinet with the frequency and modulation controls mounted on the front panel. Connections for power, stimulus output, and sync output are located on the rear panel of the cabinet.

The ICL8038 is connected for minimum distortion as outlined in the INTERSIL Data Book (Intersil, Inc., 1979), with one exception. Since, in our application, we have no need of duty cycles other than $50 \%$, pins 4 and 5 are tied together. In this configuration, variable resistor $\mathbf{R a}$ in series between these pins and $\mathrm{V}+$ controls the frequency of oscillation. The frequency of oscillation is also set by the value of $\mathrm{C} 1$, with the frequency being described by the equation: frequency $=0.15 /(\mathrm{Ra} \times \mathrm{C} 1)$. Instead of using single potentiometers for $\mathrm{Ra}, \mathrm{Rb}$, and $\mathrm{Rc}$, one may connect a number of fixed (or variable) resistors in a switching arrangement at each of these locations to permit selection of the resistor(s) appropriate for the task at hand. Our prototype unit utilizes rotary switches to select 12 different variable resistors for $\mathrm{Ra}$ and 10 different variable resistor pairs for $R b$ and $R c$. We adjust the $R b / R c$ pairs to provide signals with different modulations but with identical average levels. R1 and $R 2$ provide some control of the symmetry and the duty cycle of the sine wave generator.

Because the square wave output of the ICL8038 (pin 9) is not committed to a fixed level, the voltage swing may be adjusted to meet the requirements of most applications. In our lab, this line is tied to $+5 \mathrm{~V}$ through an external $330-\Omega$ resistor for use as a TTL-compatible trigger for our averaging computer. The maximum operating voltage for both IC1 and IC2 is $+15 \mathrm{~V}$. 


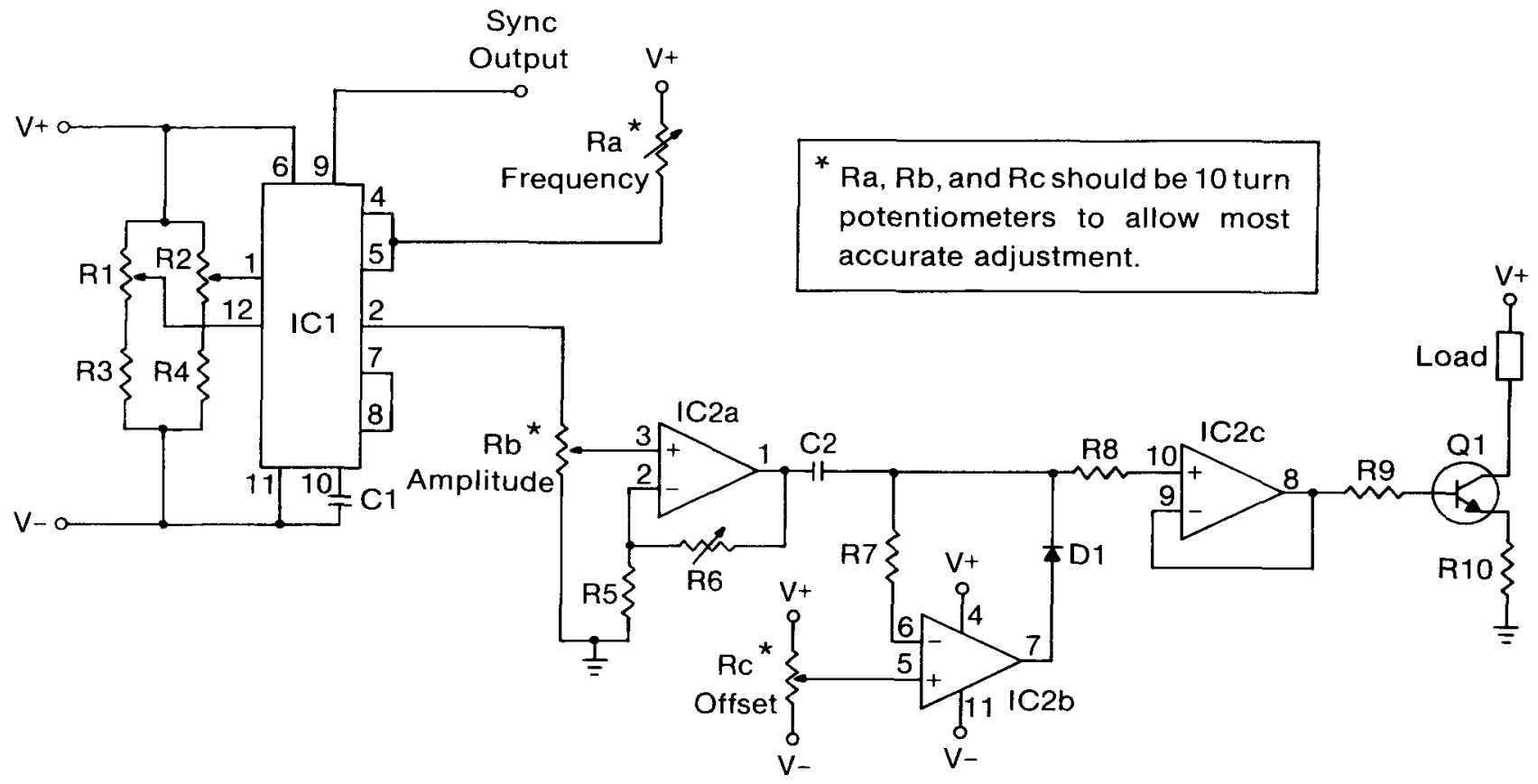

Figure 1. Schematic diagram of stimulus waveform generator. The $V+$ and $V-$ sources can range from 5 to $15 \mathrm{~V}$ but should always be equal (e.g., $\mathrm{V}+=+10 \mathrm{~V}$ and $\mathrm{V}-=-10 \mathrm{~V}$ ). Note that the fourth section of the LM124 op amp (IC2d) is not used and is therefore available for circuit modifications or enhancements.

The electrical characteristics of transistor Q1 are not critical. The only factors to bear in mind during selection are the current-handling capability of the transistor and the requirements of the load. Be sure that resistor R10 in the emitter circuit is rated at a wattage sufficient for your purposes. Consider, for example, the LED array used in our prototype, which draws approximately 1.5 A (maximum) from a 5-V source. Approximately $1.2 \mathrm{~V}$ is dropped across the LED array, and approximately another $1.4 \mathrm{~V}$ is dropped across $\mathrm{Q} 1$. This leaves $2.4 \mathrm{~V}$ across $\mathrm{R} 10$. Therefore, the power dissipated in this resistor is: $2.4 \mathrm{~V} \times 1.5 \mathrm{~A}=3.6 \mathrm{~W}$. On the basis of this calculation and conservative design criteria, R10 should be rated at a minimum of $5 \mathrm{~W}$.

Alignment of the stimulus waveform generator is straightforward. First, adjust R1 and R2 for minimum distortion of the sine wave at pin 2 of ICl as viewed on an oscilloscope. Next, again use an oscilloscope to ad-

Table 1

\begin{tabular}{|c|c|c|c|}
\hline Quantity & Part No. & Description & $\begin{array}{c}\text { Radio Shack } \\
\text { Part No. }\end{array}$ \\
\hline 1 & $\mathrm{C} 1$ & $10-\mu \mathrm{F}$ capacitor & $272-999$ \\
\hline 1 & $\mathrm{C} 2$ & 4.7- $\mathrm{F}$ capacitor & 272-998 \\
\hline 1 & D1 & 1N34A germanium diode & $276-1123$ \\
\hline 1 & IC1 & ICL8038 function generator & $276-2334$ \\
\hline 1 & IC2 & LM124 (324) op amp & $276-1711$ \\
\hline 1 & Q1 & 2N3055 NPN power transistor & 276-2041 \\
\hline 2 & $\mathbf{R} 1, \mathbf{R} 2$ & $100 \mathrm{~K} 10$-turn potentiometers & $\left(43 \mathrm{P}-100 \mathrm{~K}^{*}\right)$ \\
\hline 3 & R3,R4,R7 & $10 \mathrm{~K} 5 \%$ resistors & $271-1335$ \\
\hline 1 & R5 & $4.7 \mathrm{~K}$ resistor & 271-1330 \\
\hline 1 & R6 & $10 \mathrm{~K}$ potentiometer & 271-218 \\
\hline 1 & R8 & $2.2 \mathrm{~K} 5 \%$ resistor & 271-1325 \\
\hline 1 & R9 & 10- $\mathrm{n}$ resistor & 271-132** \\
\hline **** & $\mathbf{R a}-\mathbf{R c}$ & 10K 10-turn potentiometers & 276-1394 \\
\hline 1 & & Perforated board (cut to size) & 276-1394 \\
\hline $1 \mathrm{pkg}$ & & Push-in terminals & $270-1392$ \\
\hline 2 & & 14-pin wire-wrap DIP sockets & 276-1993 \\
\hline 1 & & 6-position dual-row barrier strip & $274-659$ \\
\hline 1 & & Instrument cabinet & $270-253$ \\
\hline
\end{tabular}

*Jameco Electronics, 1355 Shoreway Rd. Belmont, CA 94002. $10-\Omega 10-W$ resistors that can be paralleled to obtain $5 \Omega$. 
just R6 for the required signal amplitude at pin 1 of IC2a. Factors such as power supply voltage and load requirements determine this setting. In our application, a 3-V peak-to-peak signal at the output of IC2a works best. Finally, adjust $R a, R b$, and $R c$ to meet your frequency, modulation, and level requirements.

A parts list for this device is shown in Table 1. It was our intent to construct this unit with a minimum number of readily available parts. The total cost for this device is in the $\$ 30.00$ range. Use of switch-selected resistors for $R a, R b$, and $R c$ will raise the cost somewhat, as will a power supply if one is not already available. A well- regulated power source is necessary to ensure stable waveform parameters.

\section{REFERENCES}

INTERSIL, INC. (1979). INTERSIL data book (pp. 5-267). Cupertino, CA: Author.

National Semiconductor Corporation. (1982). Linear databook (pp. 3-172). Santa Clara, CA: Author.

(Manuscript accepted for publication June 28, 1984.) 